\title{
L'évolution de la place des femmes en agriculture au prisme des rapports familiaux de production
}

Sabrina Dahache ${ }^{1}$

\section{Introduction}

Les exploitations agricoles familiales se sont historiquement constituées en référence au modèle de la complémentarité entre les femmes et les hommes (Barthez, 1982). L'ethos professionnel légitimait la captation par les hommes du statut de chef d'exploitation et les rôles sexués assignés aux femmes : " collaboratrices du mari ", " maîtresses de maison » et " mères ménagères et éducatives " (Lagrave, 1987). Les rapports sociaux de production reposaient sur des relations hiérarchiques entre les sexes, les générations et les catégories socioprofessionnelles. L'organisation du travail agricole était basée sur une division sexuée des activités productives. Cette modalité contribuait à tracer les frontières entre les femmes et les hommes, en naturalisant les compétences associées à leurs fonctions et leurs statuts.

À partir des années 1980, les modernisations organisationnelles et techniques de l'agriculture (agrandissement des structures, externalisation ou délégation du travail, motorisation et mécanisation permettant l'augmentation de la productivité des productions animales, viticoles, etc.) ont entraîné des transformations importantes. Au-delà des phénomènes de désaffectation féminine, l'éclatement des systèmes d'exploitation a laissé place à de nouvelles formes d'agriculture qui ont recomposé les rapports sociaux de production, ainsi que les représentations sociales relatives à l'équation : hommes $=$ production et femmes $=$ travail dans l'ombre . L'individualisation $\mathrm{du}$ travail comme aspiration individuelle et comme injonction professionnelle (Cardon et al., 2009) s'est déployée au sein des entreprises agricoles modernisées. L'autonomie des femmes par rapport au système patriarcal est passée par plusieurs canaux. Des générations de filles et d'épouses d'agriculteurs ont développé des revendications propres visant l'accès à un travail émancipateur, une indépendance économique et plus largement une reconnaissance professionnelle (Lagrave, 1987).

1. Université de Toulouse II, UMR Dynamiques Rurales, 31058 Toulouse Cedex 9, France, dahache@univ-tlse2.fr

Ce chapitre de l'ouvrage L'agriculture en famille : travailler, réinventer, transmettre est publié en Open Access sous licence creative commons CC-BY-NC-ND permettant l'utilisation non commerciale, la distribution, la reproduction du texte, sur n'importe quel support, à condition de citer la source. 
Ces revendications ont rendu possible des évolutions significatives en matière de droits professionnels et sociaux, mais aussi des transformations juridiques et sociologiques de leur statut et de leur place dans «l'institution de l'exploitation familiale " (Rémy, 2013) ${ }^{2}$. Elles ont conduit à une remise en cause des modèles fondés sur une hiérarchie qui s'opérait au niveau des rapports familiaux de travail, au niveau des catégorisations professionnelles sexuées et au niveau des identités féminines et masculines. Les processus de dissociation entre ménage et unité de l'entreprise agricole participent de ces bouleversements. Ils cohabitent avec les déplacements de la division sexuelle du travail et la domination des référentiels managériaux masculins. Les actions organisationnelles dans les entreprises modernisées abritent des rationalités plurielles où s'affirment des dynamiques professionnelles individuelles et collectives qui ne sont pas sans rappeler les vieux schémas que l'on aurait pu penser dépassés. Elles apparaissent comme de nouvelles formes de contrainte lorsque les femmes exercent leur métier sous la "tutelle maritale" (Rieu, 2005). Dans le même temps, ces entreprises constituent des construits sociaux bâtis autour de projets d'émancipation individuels et collectifs. Les spécialisations professionnelles s'appuient sur des collectifs de travail de différentes natures et formes, structurés par les nouvelles configurations des différents rapports sociaux de production. Les dispositifs organisationnels impliquent des arrangements multiples privilégiant des dimensions individuelles et collectives de l'émancipation au travail.

Cette contribution vise à montrer comment les formes d'organisation et de division du travail contribuent à conformer la place des femmes dans les entreprises agricoles familiales. Nous proposons d'étudier la dialectique du travail individuel et du travail collectif comme une dynamique mettant en jeu des contradictions entre les assignations professionnelles, via notamment la division sexuée du travail, et la nécessité pour les femmes de s'en éloigner pour asseoir leur légitimité. Notre hypothèse consiste à penser que leur inscription dans les logiques collectives d'autonomisation professionnelle dépend des systèmes complexes d'organisation du travail comme résultat des différents rapports sociaux de production, à la fois comme contrainte et support d'émancipation. Dans quelle mesure les exploitations familiales peuventelles permettre d'organiser de nouvelles formes de rapport au travail agricole pour les femmes? En quoi la réappropriation par les femmes des logiques d'organisation du travail (polyvalence et/ou spécialisation, modulation des temporalités professionnelles associées aux systèmes d'activité, pratiques de mobilité entre ateliers et

2. À titre d'exemples : la loi d'orientation agricole du 4 juillet 1980 instaure les statuts de conjointe co-exploitante et de conjoint collaborateur. En 1985, une nouvelle mesure autorise les couples à constituer une EARL (exploitation agricole à responsabilité limitée), sans une tierce personne. Les femmes peuvent désormais accéder au statut de chef d'exploitation. Le décret du 23 février 1988 permet aux deux époux de bénéficier des aides à l'installation (dotation jeune agriculteur, emprunts bonifiés, etc.). Plus récemment, en 1999, la nouvelle disposition du statut de conjoint collaborateur offre aux agricultrices des droits supplémentaires en matière de retraite et certaines prestations sociales. Depuis 2005, ce statut est rendu accessible sans l'autorisation préalable du chef d'exploitation. Enfin, en 2010, est supprimée l'interdiction de constituer un GAEC (groupement agricole d'exploitation en commun) entre conjoints. 
productions, etc.) des exploitations familiales participe-t-elle au "processus d'individualisation et au processus collectif d'émancipation " (Cardon et al. 2009) ? Les tensions autour du travail agricole et de ses divisions les éloignent-elles des possibilités de reconnaissance et d'autonomie ? Pour mener à bien cette analyse, on s'intéressera, dans un premier temps, aux effets des mutations structurelles sur la place des femmes dans le système exploitation/famille. L'articulation de ces deux réalités permettra de mieux comprendre le rôle qu'elles jouent dans les modalités de ces changements. Dans un second temps, on tentera d'analyser les situations de travail individualisées, isolées et collectives, à partir d'une entrée par les rapports de genre mis en lien avec les différents rapports sociaux de production. Enfin, nous prêterons une attention à l'articulation entre travail professionnel et travail domestique dans les processus de construction et de transformation des normes et des pratiques des femmes.

\section{Précisions méthodologiques}

Nous nous appuyons sur un ensemble de recherches menées dans douze régions en France $^{3}$ dans le cadre d'un doctorat sur la mixité de l'enseignement agricole (Dahache, 2012) et d'une recherche post-doctorale consacrée à l'évaluation des dispositifs d'action publique qui donnent la mesure des enjeux de l'insertion des jeunes dans les entreprises agricoles et rurales (Dahache, 2013 a et b ; 2014). Sans s'en tenir aux différentiels de formation, de reconnaissance des compétences et d'accès aux professions agricoles et rurales, la thèse consistait à analyser l'impact des changements qui affectent les territoires ruraux sur la division sexuelle formation-qualificationtravail, mais aussi les effets de la mixité de l'enseignement agricole et des professions auxquelles il prépare (agriculteur, horticulteur, viticulteur, technicien agricole, etc.). Les analyses ont été poursuivies dans le cadre du post-doctorat. Elles attirent l'attention sur l'individualisation des parcours accompagnée d'une démocratisation des pratiques, mais également sur les capacités des nouvelles générations à infléchir les processus sociaux qui les dépassent.

Dans ce chapitre, la confrontation des résultats vise à croiser les données empiriques. Nous avons choisi de porter nos observations sur les systèmes de travail et de gestion vie professionnelle/vie familiale qui prennent la forme d'une organisation (négociation, délégation, etc.). Ce choix, qui peut paraître réducteur, a été effectué pour pointer les tensions entre les recompositions des rapports sociaux de production et les diverses formes sexuées d'organisation du travail. Les analyses secondaires nous semblent restituer de manière vivante la diversité des conditions d'exercice et de travail des jeunes récemment installés en agriculture. Elles reposent sur des approches comparatives et des méthodologies qualitatives et quantitatives : 75 entretiens biographiques réalisés auprès d'hommes et de femmes qui exercent dans des structures

3. Les enquêtes ont été réalisées dans les régions suivantes : Auvergne, Aquitaine, BasseNormandie, Bretagne, Centre, Champagne-Ardenne, Corse, Guadeloupe, Haute-Normandie, Languedoc-Roussillon, Midi-Pyrénées et Poitou-Charentes. 
individuelles et collectives ; 205 questionnaires passés aux anciens élèves des formations de la production ; et des observations ethnographiques lors d'assemblées générales de groupements de vulgarisation agricole, de coopératives de production, de syndicats agricoles, de manifestations agricoles organisées autour des questions sur la place des femmes en agriculture, mais aussi dans de nombreuses entreprises agricoles. Âgées de vingt à trente-sept ans, les personnes enquêtées exercent la profession agricole depuis plus d'un an. Certaines ont occupé différents statuts d'emploi avant leur installation (technicien ou salarié agricole, conseiller-clientèle, technico-commercial, assistant d'éducation, etc.). Une majorité exerce dans une entreprise sociétaire. Titulaires d'un diplôme agricole (baccalauréat professionnel "Conduite et gestion d'une exploitation agricole ", brevet de technicien supérieur agricole " Analyse et conduite des systèmes d'exploitation agricole », etc.), elles bénéficient des statuts de chef d'exploitation et d'associé exploitant.

\section{Effets des mutations structurelles sur la place des femmes dans le système exploitation/famille}

L'institutionnalisation de l'exploitation agricole familiale se confond avec l'apparition d'une nouvelle politique agricole orientée vers la modernisation des dispositifs de production (Muller et al., 1989). Les lois d'orientation agricole de 1960 et 1962 ont défini des normes d'interventions publiques fondées sur la promotion d'une « exploitation à deux UTH» (Unités de travail-homme) susceptible d'appliquer les méthodes techniques modernes et de permettre le plein-emploi du travail (Rémy, 2013). Les réformes successives ont confirmé le rôle de la famille qui sous-tend les dynamiques professionnelles agricoles. Depuis les années 1980, les configurations des systèmes d'exploitation ont fait l'objet de nombreux ajustements appropriés aux réalités économiques, sociales et environnementales. L'essor des formes sociétaires participe à ces bouleversements, en impulsant une dissociation du travail agricole et des capitaux de production d'une part, et des logiques patrimoniales et foncières d'autre part (Hervieu et Purseigle, 2013). Les EARL représentent 15,3\% des exploitations en 2010, contre $8 \%$ en 2000. Les GAEC formaient 5,9\% en 2000 pour atteindre 7,2 \% en 2010 (Agreste - Recensement agricole, 2012).

Les restructurations des dispositifs d'exploitation s'inscrivent dans le sens d'une nouvelle forme de mobilisation et d'organisation des collectifs de travail. Elles ont entraîné des mutations économiques et démographiques qui sont à l'origine de certaines transformations structurelles. En effet, les études statistiques font état de deux phénomènes contradictoires concernant la place des femmes dans l'agriculture $^{4}$ - la désaffectation féminine envers les entreprises agricoles et la féminisation des groupes professionnels des chefs d'exploitation, d'associés d'une société agricole et de salariés de la production.

4. Les analyses sur la féminisation des groupes professionnels de l'agriculture et les orientations productives s’appuient sur les études statistiques réalisées par la mutualité sociale agricole (2012). 


\section{Des conjointes qui exercent plutôt en dehors des entreprises agricoles}

Comme dans les autres groupes professionnels d'indépendants, de plus en plus de conjointes s'investissent dans des carrières professionnelles en dehors des entreprises familiales. La part des épouses d'exploitants qui exercent une autre profession est passée de $23 \%$ en 1979 à $47 \%$ en 2003 (Rattin, 2006). Les moins de 25 ans représentent $75 \%$, les 30-34 ans $68 \%$, les 40-44 ans $47 \%$ et les 50-54 ans 30\%. Cette désaffectation féminine témoigne d'un important écart entre les intentions ayant présidé à la promotion du modèle de "l'exploitation à deux UTH » et les comportements des femmes face à leurs emplois et leurs carrières. Elle correspond à de multiples logiques sociales au regard des effets de genre ${ }^{5}$ et de génération, ainsi que de "l'ouverture conjugale et du décentrement de la vie privée " (Giraud et Rémy, 2008). La division sexuée du travail évolue dans le sens d'une multiplication des couples à double carrière. Cela implique des mobilisations professionnelles et des stratégies de gestion des carrières pour chacun des conjoints. Auparavant, la pérennité des exploitations impliquait un fort investissement. Elle mobilisait les ressources de l'ensemble du groupe familial et engendrait une délégation du travail aux femmes. L'individualisation des activités et des carrières professionnelles dans les couples modifie l'équation professionnelle et familiale de l'agriculture où le travail professionnel et le travail domestique des femmes ne constituaient qu'un seul et même travail, celui de l'épouse : affaire de différenciation et de complémentarité.

Concomitamment à ces phénomènes de désaffectation féminine dans les entreprises agricoles, la proportion des conjointes actives diminue depuis une dizaine d'années. Elles atteignent 41326 en 2010. Parmi elles, $14 \%$ sont des collaboratrices à titre secondaire et $86 \%$ à titre principal. Ces femmes exercent dans des sociétés agricoles, en viticulture d'appellation, en grandes cultures ou dans des entreprises orientées vers l'élevage de porcins et de volailles. Autant d'orientations qui ont par ailleurs recours à une main-d'œuvre salariée. Alors que les collaboratrices à titre principal effectuent en moyenne $70 \%$ d'un plein temps, les collaboratrices à titre secondaire travaillent à mi-temps et occupent en parallèle d'autres statuts d'emploi pour prendre de la distance avec leur position subalterne dans le système exploitation/ famille (MSA, 2012).

\section{Une féminisation des groupes professionnels de l'agriculture}

Ces évolutions marquent un décalage avec le constat d'une féminisation des groupes professionnels des salariés de la production, des associés d'une société agricole et des chefs d'exploitation. Entre 1988 et 2010, la part des salariées de la production agricole a plus que doublé. Elles représentaient 15,7 \% en 1988, pour atteindre

5. Le concept de rapports de genre renvoie aux tensions entre le groupe social des hommes et celui des femmes autour des enjeux du travail. Par genre, nous entendons le processus de construction sociale des hiérarchies et des différences sexuées (Kergoat, 2000). 
$38 \%{ }^{6}$ en 2010. La féminisation des groupes professionnels des chefs d'exploitation et d'associés exploitants n'est certes pas de la même ampleur : 14,7\% en 1988 et 24,1 \% en 2010 (Tab. 1). Mais, elle reflète une distanciation des femmes à l'égard du modèle des conjointes collaboratrices.

Tab. 1. Évolution des chefs d'exploitation et associés exploitants, 1988 à 2010.

\begin{tabular}{|ccccccc}
\hline & $\mathbf{1 9 8 8}$ & $\mathbf{1 9 9 0}$ & $\mathbf{2 0 0 1}$ & $\mathbf{2 0 0 6}$ & $\mathbf{2 0 0 8}$ & $\mathbf{2 0 1 0}$ \\
\hline Femmes en \% & 14,7 & 16,9 & 23,9 & 24,2 & 24,5 & 24,1 \\
\hline Hommes en \% & 85,3 & 83,1 & 76,1 & 75,8 & 75,5 & 75,9 \\
\hline Ensemble $\left(\boldsymbol{n}^{\text {bre }}\right)$ & $\mathbf{1 0 1 6 8 0 0}$ & $\mathbf{9 2 3 0 0 0}$ & $\mathbf{5 8 2 7 1 7}$ & $\mathbf{5 3 4 8 9 1}$ & $\mathbf{5 1 3 6 1 5}$ & $\mathbf{4 9 6 3 6 4}$ \\
\hline
\end{tabular}

Sources : CCMSA, 2001 et 2012. Calculs effectués par l'auteure.

En 2011, plus de la moitié des femmes chefs d'exploitation exercent dans des sociétés agricoles. Parmi ces installations sous forme sociétaire, on compte $17 \%$ d'associées de GAEC (83 \% pour les hommes). $23 \%$ des chefs d'exploitation installés en EARL sont des femmes. Les exploitantes situées dans la tranche d'âge des 40-45 ans privilégient les formes sociétaires (45-49 ans pour les hommes). Quel que soit leur âge, les hommes sont sur-représentés parmi les chefs d'exploitation installés à titre individuel ( $77,5 \%$ contre $22,5 \%$ pour les femmes). Au-delà des 55 ans, les femmes sont près de 8200 dans les GAEC et 6700 dans les EARL. L'orientation des plus jeunes vers l'exercice en société conforte une tendance à l'homogénéisation des modes d'exercice des hommes et des femmes (Tab. 2) : 26,7\% des femmes âgées de moins de 40 ans sont installées en individuel (soit $45 \%$ des effectifs des exploitantes), 38,9\% en EARL (28,4\% des femmes) et 20,1\% en GAEC (14,5\% des femmes).

Tab. 2. Les nouvelles installations selon les formes juridiques en 2011.

\begin{tabular}{lcccc}
\hline & \multicolumn{2}{c}{$\mathbf{4 0}$ ans et moins } & \multicolumn{2}{c}{ Plus de $\mathbf{4 0}$ ans } \\
\hline En individuel & Hommes en \% & Femmes en \% & Hommes en \% & Femmes en \% \\
GAEC & 73,3 & 26,7 & 27 & 73 \\
EARL & 79,9 & 20,1 & 7,1 & 92,9 \\
Autres & 61,1 & 38,9 & 17,9 & 82,1 \\
\hline Ensemble $\left(\boldsymbol{n}^{\text {bre }}\right)$ & 64 & 36 & 35,6 & 64,4 \\
\hline
\end{tabular}

Sources: CCMSA, 2012. Calculs effectués par l'auteure.

6. Ces données statistiques concernent toutes les catégories du salariat de la production agricole, y compris familial (mutualité sociale agricole, 2012). 
Ces processus de féminisation s'inscrivent dans une configuration de genre en mutation qui place les femmes face à des bénéfices potentiels : possibilités d'accès aux formations qualifiantes et aux aides à l'installation, possibilités de constituer une EARL ou un GAEC entre époux et autres perspectives d'emploi dans l'agriculture. Les agricultrices semblent également tirer toutes les conséquences des avancées concernant leur statut juridique dans la profession agricole. Leur inscription dans les dispositifs sociétaires constitue l'une des évolutions majeures de ce secteur depuis quelques années dans le sens où elle formalise la séparation de la sphère productive et de l'espace familial.

\section{Féminisation et diversification professionnelle}

Ces avancées relatives aux modes d'exercice professionnel des femmes en agriculture apparaissent comme un indice de nouvelles dynamiques professionnelles lorsque l'on considère les orientations productives. Les salariées de la production agricole exercent majoritairement dans les domaines de la production viticole (39\%), des cultures spécialisées (33\%) et de l'élevage non spécialisé (16\%). Les données disponibles révèlent une convergence des contenus d'activité des femmes et des hommes situés dans ce groupe professionnel.

De leur côté, les femmes chefs d'exploitation et les associées d'une société agricole s'orientent davantage vers l'élevage de $\operatorname{chevaux}^{7}(44,2 \%)$, de volailles et de lapins $(35,3 \%)$, d'ovins et de caprins $(31,1 \%)$. Elles sont moins représentées dans l'élevage de bovins $(22,7 \%)$ et sont encore plus rares parmi les producteurs de bois $(1,8 \%)$ et les entrepreneurs de travaux agricoles ( $8 \%)$. Les statistiques sur les nouvelles installations révèlent les transformations des systèmes de production (Tab. 3). Ceci induit des déplacements des ségrégations sexuelles des activités productives et l'apparition de spécialisations professionnelles différenciées chez les femmes autour des activités de loisirs $(62,6 \%)$ et de soins aux productions animales $(61,6 \%)$. Les activités prédominantes des hommes demeurent inchangées : cultures céréalières $(54,6 \%)$, cultures légumineuses $(52,7 \%)$, cultures spécialisées $(56,7 \%)$ et élevage de bovins $(54,9 \%)$. Les femmes étant légèrement plus représentées dans les secteurs de la viticulture $(52,1 \%)$, de l'élevage de vaches laitières $(52,4 \%)$, et de l'élevage d'équidés ${ }^{8}(52,3 \%)$. Elles tendent donc à impulser un mouvement de diversification, marqueur de nouvelles formes de différenciation sexuée.

7. L'élevage de chevaux correspond à plusieurs activités selon l'utilisation à laquelle ils sont destinés : le loisir, la compétition ou la boucherie.

8. L'élevage d'équidés (chevaux, ânes, poneys, etc.) renvoie également à plusieurs pôles d'activités : activités de production et de valorisation (élevage, entraînement, compétition); commercialisation des produits (viande, cuir, lait de jument ou lait d'ânesse) ; services (pensions, soins aux équins...) ; activités de sport, de loisir ou de travail. 
Tab. 3. Les mentions de spécialisation dans les nouvelles installations en 2011.

\begin{tabular}{|lcc|}
\hline \multicolumn{1}{c}{ Mentions de spécialisation } & Hommes en \% & Femmes en \% \\
\hline Cultures céréalières & 54,6 & 45,4 \\
\hline Cultures légumineuses & 52,7 & 47,3 \\
\hline Production viticole & 47,9 & 52,1 \\
\hline Élevage de bovins & 54,9 & 45,1 \\
\hline Élevage d'équidés & 47,7 & 52,3 \\
\hline Élevage d'ovins et de caprins & 51 & 49 \\
\hline Élevage de volailles & 47,4 & 52,6 \\
\hline Activités de loisirs & 37,4 & 62,6 \\
\hline Activités de soins aux productions animales & 38,2 & 61,6 \\
\hline
\end{tabular}

Sources : CCMSA, 2012. Calculs effectués par l'auteure.

Les redéfinitions des référentiels de profession (statuts, modalités de professionnalisation, configuration juridique des systèmes d'exploitation, etc.) ont permis l'ouverture d'espaces de négociation dans les entreprises modernisées (GAEC, EARL, etc.), ainsi qu'une reconnaissance des divers registres des pratiques non agricoles autrefois dévalorisées. Ces phénomènes ont constitué un levier déterminant dans la transformation du rapport des femmes à la profession agricole. Léclatement et l'individualisation des temps et du travail agricole accompagnent ces évolutions. Il s'agit à présent d'examiner, en détail, les différentes formes sexuées d'organisation du travail pour comprendre en quoi elles participent à légitimer les principes de différenciation sexuée ou bien à les dépasser.

\section{Les configurations sexuées des systèmes d'activité dans les exploitations}

Les dispositifs organisationnels et techniques des entreprises agricoles induisent une individualisation du travail qui peut configurer ou limiter les passages à des pratiques collectives. Ces dynamiques ne sont pas déterminées, ni posées dans les mêmes conditions selon les configurations des exploitations familiales (composition des collectifs de travail, configuration des systèmes d'activité, etc.), les aspirations individuelles et collectives, mais également la dynamique des rapports de travail, etc. (Dufour et Giraud, 2012). Nos différentes recherches permettent d'identifier trois profils de jeunes femmes situées dans des cadres organisationnels différenciés?

9. Pour élaborer ces profils, plusieurs indicateurs ont été pris en compte : les formes d'organisation et de division du travail, les modes de gestion des temporalités, les contenus d'activité, les 
Ces profils témoignent de la complexité des enjeux sexués de l'organisation du travail agricole et de ses divisions.

\section{Des dispositifs organisationnels qui articulent travail individuel et travail collectif}

Le premier profil regroupe des jeunes exploitantes dont les expériences au sein des entreprises agricoles sont caractérisées par une légitimité forte accordée à la disponibilité permanente dans le travail agricole, permettant un cloisonnement des sphères professionnelles et familiales. Dans leur famille, elles ont été initiées aux pratiques agricoles. La mobilisation de leurs parents autour de leurs plans de professionnalisation en agriculture leur a servi d'assise pour élaborer des projets qui dérogent aux attendus. Ainsi, elles ont construit leur identité professionnelle en se démarquant du modèle féminin traditionnel. Les exploitations au sein desquelles elles exercent (EARL ou GAEC composés de deux à dix membres) associent aux productions céréalières (maïs, tabac, blé, etc.) plusieurs productions animales (élevage de veaux ou d'ovins et élevage de volailles; gavage et élevage de palmipèdes gras ou de volailles). Disposant d'une équipe de travail (salariés agricoles), leurs logiques managériales articulent travail collectif et travail individuel pour accroittre le potentiel productif de leurs structures. Elles reposent sur des règles visant à organiser leur système d'activité. Ces règles définissent les contributions individuelles et collectives, mais également leurs propres orientations techniques :

"On est partout... On fait la même chose en fait. Le gavage, on commence à six heures [...]. L'élevage, on s'en occupe, on fait tout. On alterne quand il faut passer dans les parcs. Le reste, soit on y va tous, soit on y va seul ${ }^{10}$.

Les dispositifs organisationnels qu' elles accumulent structurent la conduite des activités agricoles, en jouant sur les flexibilités sociales et techniques pour une meilleure adaptation aux contraintes professionnelles (climat, absentéisme des salariés agricoles, etc.). Les arbitrages qu'elles opèrent sont orientés par leur capacité à mobiliser et à combiner différents registres d'action. Les ordres de travail de ces jeunes exploitantes ne s'inscrivent pas dans une représentation normative de la division sexuelle du travail qui découle des rapports sociaux de production entre les sexes et les générations :

"Viticulture, c'est un métier extrêmement noble qui regorge de richesse; mais qui a des valeurs que les gens ne voient pas. Il faut être dedans pour les voir. Il y a le fait de se surpasser et aller au-delà de ses capacités. On est face à des conditions climatiques extrêmes le froid, la chaleur. Et, je pense qu'il faut un mental, un physique. Et, je pense

caractéristiques des exploitations (orientation productive, salariat agricole, etc.), les responsabilisations et mobilisations professionnelles, etc.

10. Emma, 27 ans, origine agricole, installée en 2008 dans une EARL : céréales, gavage et élevage de palmipèdes gras, collectif de travail : son conjoint, un collègue et une main-d'œuvre salariale ponctuelle. 
beaucoup d'amour du métier pour le faire. Donc, moi, je sais. Je me suis dirigée làdedans parce que mes beaux-parents sont exploitants viticoles, enfin, mon beau-père, ma belle-mère non. Et, donc, je suis allée pour l'aider à la base. Après, j’étais bien dans les vignes. J'ai appris. J'ai vu quelque chose qui me ressemblait. Mais, je ne voulais pas être sous ses ordres. Je ne voulais pas être dévalorisée parce qu'on a souvent le dilemme avec des gens qui sont chez eux, qui vont se retrouver en famille. Et, bon, moi, c'est facile de se mettre à aider quelqu'un. Mais, je voulais vraiment être dans mon métier pour gérer tout ${ }^{11}$.

Les principes de l'interchangeabilité des compétences professionnelles associées au masculin et au féminin gouvernent les pratiques agricoles effectuées par les femmes. Cette modalité marque leur distanciation à l'égard des attributs et des stéréotypes de sexe. Elle passe par leur refus d'une valorisation différentielle des dispositions de genre. Engagées dans des activités diverses, ces jeunes agricultrices incarnent finalement une " maîtrise professionnelle " qui transforme les rapports de travail structurés par le genre. Cette maîtrise professionnelle leur permet d'impulser de nouvelles dynamiques. L'individualisation de leur travail associée à des passages aux pratiques collectives acquiert toute sa portée émancipatrice. Leur autonomie professionnelle tend à produire une indifférenciation des aspirations et des pratiques agricoles des deux sexes. Leurs manières de gérer les travaux agricoles (gavage, distribution des aliments dans les élevages hors-sol, gestion des activités collectives : livraisons animales, activités de vaccination, élaboration des plannings des salariés, etc.) prêtent main-forte aux logiques organisationnelles collectives. Dans les jeux coopératifs, elles disposent de ressources multiples pour gérer les relations inter et intra-professionnelles. Leur co-participation au processus de rationalisation leur permet d'améliorer les systèmes de travail et les fonctionnalités des exploitations. D'une part, elle conforte leur capacité à contrôler les ensembles organisationnels et techniques. D'autre part, elle autorise une plus grande visibilité sur la nature et les modalités de leurs propres manières d'exercer. Les configurations de leurs conditions de travail sont marquées par une intégration indifférenciée des femmes et des hommes dans les entreprises. C'est ainsi qu'elles parviennent à s'affranchir des tensions contradictoires qui sous-tendent les logiques collectives entrepreneuriales :

"Je me mêle de tout, justement, voilà, pour que ce soit dans tout, pour que je puisse au moins m'épanouir professionnellement. Et, puis, je trouve que c'est intéressant plutôt de participer à tout. Ici, il n'y a pas que les vignes. Il y a aussi le vin. Il y a le chai. Il y a les vendanges. Il y a tout ça. Donc euh, voilà moi, je veux faire évoluer les choses " 12 .

11. Solange, 26 ans, parents : fonctionnaires, installée en 2010 dans une EARL, collectif de travail : son beau-père et une main-d'œuvre salariale.

12. Anna, 32 ans, origine agricole, installée en 2007 dans une EARL, collectif de travail : son beau-père, son conjoint et une main-d'œuvre salariale ponctuelle. 


\section{Des systèmes organisationnels qui opèrent une division sexuée du travail}

Le deuxième profil est composé de jeunes femmes qui exercent dans des structures d'exploitation (EARL, SCEA ou GAEC composés de deux à cinq membres) conformées par une division sexuelle des travaux individualisés, impliquant quelques passages à des pratiques collectives : production céréalière ou viticulture et entreprise de travaux agricoles pour les hommes - gavage ou élevage (caprins, veaux, etc.) et élevage de volailles pour les femmes. Socialisées à l'exercice de la profession agricole dans leur famille ou hors contexte familial, elles ont choisi très tôt de s'orienter vers une formation de la production pour éviter une perte de contrôle sur leur devenir professionnel. Elles se sont appuyées des supports d'individualisation (titres scolaires, aides à l'installation, etc.) leur permettant de se tailler une place dans les exploitations. Ces jeunes agricultrices s'inscrivent dans des logiques professionnelles singulières qui trouvent des points d'appui dans la présence d'une main-d'œuvre familiale et/ou salariale. Leur forte implication professionnelle leur permet d'exploiter au mieux la liberté que leur procurent leurs dispositifs professionnels : délégation d'un certain nombre de tâches (entretien des bâtiments agricoles, production végétale), recentrage sur les activités des systèmes de production animale, etc. Outre leur investissement dans des travaux collectifs (déplacement des animaux, accueil des lots, etc.), ces exploitantes se ménagent généralement du temps pour des tâches qui leur offrent une certaine flexibilité (distribution du lait et des aliments sur des tranches horaires qui varient en fonction des autres activités : 4h30-7h30 ou 6h30-8h30/17h-20h ou 19h-22h, décalage des soins aux animaux, etc.). Ceci ne les affranchit nullement des dynamiques organisationnelles à mettre en œuvre au quotidien :

"On reçoit les veaux, il faut être deux au moins... Laver le bâtiment, pareil, on fait venir le jeune que l'on emploie [...]. Les veaux, oui, le matin et le soir, trois heures... Enfin, ça dépend sill y a plus de soins à faire... Au départ... Euh... Les deux premiers mois au moins, il y a plus de travail, je fais venir F. [...]. [Comment organisez-vous votre travail aux moments des récoltes, des réunions des coopératives de production, etc. ?], je commence les veaux plus tôt [...]. La journée, je reviens au bâtiment pour voir si les veaux sont gonflés, pour les sonder ou les piquer ${ }^{13}$.

Les enjeux autour des processus de rationalisation du travail des entreprises agricoles élargissent le spectre des sollicitations qui leur sont adressées. Les dispositifs managériaux collectifs (comme, par exemple, les réunions techniques dans les GAEC) créent de nouvelles contraintes. Si les principes d'autonomie et de responsabilisation individuelle sont promus dans les entreprises, les structures de production introduisent des exigences fortes. C'est dans le cadre du fonctionnement des équipes que se dessinent les dynamiques de (re)conquête des temps et des contenus de travail. La répartition des tâches et la gestion des temporalités associées aux travaux

13. Anaïs, 25 ans, origine agricole, installée en 2008 dans une EARL : céréales, élevage de veaux et entreprise de travaux agricoles, collectif de travail : son frère, son conjoint et un salarié. 
individuels et collectifs inaugurent des tensions autour du travail et de ses divisions sexuelles. De plus, l'emploi du temps de ces jeunes femmes est calqué sur les travaux collectifs. Il repose aussi sur d'autres formes de division de l'espace et du travail : les courses pour les entreprises, les démarches et responsabilités administratives, etc. Les sollicitations multiples induisent des réajustements en réponse aux assignations professionnelles et aux pénibilités des conditions et de l'organisation des diverses activités qui leur échoient.

Les expériences professionnelles de ces exploitantes sont ainsi marquées par des tensions entre les ordres négociés de travail qui prennent sens dans les différents rapports sociaux de production. Elles revendiquent l'originalité de leur pratique du métier. Les référentiels auxquelles elles s'appuient s'inscrivent dans les représentations partagées d'une nécessaire division sexuée du travail agricole. Si elles remettent en question le modèle des femmes assignées à des "situations d'aide ", elles rejettent certaines contraintes professionnelles instaurées comme spécifiquement féminines (désengagement des processus collectifs de rationalisation, etc.). En inscrivant leurs choix professionnels en rupture avec le modèle féminin traditionnel, elles s'arrangent avec les normes en place et exercent la profession agricole à leur manière.

\section{L'isolement dans le travail agricole comme contrainte liée au genre}

"Non, non, ça ne se passe pas toujours bien. Voilà, eux, c'est des hommes. Donc, ils sont que sur les tracteurs. Il n'y a que la terre qui compte. Et, moi, il faudrait que je fasse 60 heures par semaine. Il faudrait que je sois toujours au travail : gaver, tuer, plumer tout, seule. C'est du lever du soleil jusqu'au coucher du soleil que je travaille, c'est simple. Ça, ils ne le voient pas. Ils voient que ce quils font " ${ }^{14}$.

Ce dernier profil concerne les jeunes exploitantes confrontées à des situations d'isolement dans les exploitations (EARL ou GAEC composés de deux à trois membres et autres structures d'exploitation). Après quelques détours (parcours de formation discontinus, exercice d'une autre profession, etc.), elles ont souvent dû motiver leur choix professionnel auprès de leurs parents et des professionnels de l'orientation scolaire peu familiarisés à l'agriculture. Un cloisonnement sexué des spécialisations professionnelles s'opère dans les exploitations au sein desquelles elles exercent. Il est synonyme de reproduction des rapports de genre. Les référentiels sexués autour des fonctions et des compétences professionnelles organisent la division sexuée des travaux agricoles. Les systèmes d'activité (production céréalière pour les hommes gavage, élevage de volailles et conserverie ou maraîchage et vente directe pour les femmes) constituent des éléments structurants de l'identité sexuée des acteurs des entreprises agricoles.

14. Marie, 33 ans, parents : ouvriers, installée en 2006 dans un GAEC, collectif de travail : son beau-père et son beau-frère. 
Dans ces systèmes, les jeunes femmes sont promues pour tenir des rôles complémentaires de ceux des hommes. Elles s'investissent dans des activités socialement dominées et fondées sur le maintien des stéréotypes en matière de "spécificité féminine ". Leurs situations de travail individualisées créent les conditions objectives d'une recomposition des territoires masculins et féminins. Ce processus s'accompagne de formes sexuées d'organisation des temporalités. L'usage des temps professionnels associés aux différentes activités (de production, de transformation agroalimentaire et de commercialisation) peut se révéler en jeu. Ces exploitantes ont à jongler entre les exigences de rentabilité et la gestion de leurs dispositifs professionnels. Leur charge de travail augmente en fonction des spécificités fonctionnelles de leurs structures de travail. L'éclatement et la variété des contenus et lieux d'activité conduisent à des instabilités temporelles. Ces temporalités et l'individualisation du travail agricole complexifient singulièrement leur possibilité de construire des activités et des temps collectifs de travail. Cette possibilité est d'autant plus restreinte que les faibles potentialités des entreprises (endettement, système d'exploitation de petite taille, etc.) ne leur permettent pas d'avoir recours à une main-d'œuvre salariale. Leurs modes d'organisation du travail agricole et de ses temporalités (morcellement des temps professionnels, décalages des activités, intensification des rythmes de travail lors du cumul d'activités) induisent leur mise à l'écart dès lors qu'elles travaillent non seulement seules, mais aussi en situation de travail isolée. Dans ce contexte organisationnel, l'absence d'une participation de leurs associés dans leurs dispositifs professionnels les enferme dans le " registre de l'individuel ». Ce contexte constitue un cadre de contraintes pesant sur leur insertion dans les collectifs de travail parce que " producteur de situations individualisées et individualisantes " (Lada, 2009). Il s'agit pour ces jeunes exploitantes de " tenir au travail " et non d'agir pour transformer les ordres " genrés » des activités agricoles, ainsi que les dispositifs organisationnels des entreprises instaurés par et pour les hommes :

"Parce que, moi, comme je n'étais pas du milieu, j'ai tout fait pour monter ma petite salle de gavage. Après, je livrais à un conserveur. Et, après, je me suis fait une conserverie à moi [...]. On m'a rigolé au nez en me disant: tu te débrouilles, faut que ça marche, faut que tu fasses tourner " ${ }^{15}$.

Si l'articulation travail individuel et travail collectif ouvre la voie de l'autonomie pour les femmes, ces avancées se conjuguent avec les configurations traditionnelles : l'absence de coopérations contribue à l'activation des fondements de leur marginalisation sociale dans la profession agricole.

15. Lisa, 35 ans, père : artisan et mère : aide-ménagère, installée en 2000 dans un GAEC : céréales, vignes, gavage, conserverie, collectif de travail : son beau-père et son conjoint (mi-temps). 


\section{Des réajustements dans la sphère domestique et familiale}

Les conditions d'exercice des jeunes exploitantes dans les structures collectives sont à l'origine des réajustements qui s'opèrent dans la sphère domestique. Leurs logiques professionnelles tout comme la nature de leurs systèmes d'activité produisent des modèles spécifiques de forte implication dans le travail. Elles induisent des stratégies de régulation des temporalités, qu'elles soient individuelles ou collectives. Ces stratégies marquent la volonté (si ce n’est la pratique réelle) de séparation entre le temps professionnel et le temps privé. Mais elles ne prennent pas la même configuration au regard des rapports de genre dans le système exploitation/famille.

\section{Une séparation des temps professionnels et domestiques qui repose sur les femmes}

Les contextes organisationnels configurés par des spécialisations professionnelles sexuées reposent souvent sur une division sexuelle des usages du temps libre. Pour les hommes, ces temps libres sont affectés à des engagements professionnels en dehors des entreprises (syndicalisme, etc.), au repos et aux loisirs. Pour les jeunes exploitantes, ils sont généralement consacrés à la gestion de la sphère domestique. Les sollicitations et les travaux multiples leur laissent peu de temps disponible. L'enchaînement des activités s'opère en fonction des temps domestiques et parentaux. Pour atteindre des conditions d'activité optimales dans la sphère productive, elles mettent en ouvre toute une série de stratégies. La gestion des différentes temporalités est continue et leur calibrage est constant. Ces modalités impliquent une organisation méticuleuse, une régulation maximale des pratiques domestiques et une redéfinition des normes revues à la baisse en termes d'exigences : ménage et repas moins élaborés, limitation des déplacements pour les courses alimentaires, etc., le but étant que cela soit réalisé le plus rapidement possible : "Quand je rentre, jattaque tout de suite parce que je ne me pose pas. Quand j'arrive, je fais le manger, une machine à laver, je range un peu. Après c'est propre ou ce n'est pas propre. C'est comme ça ${ }^{16}$.

Le recours aux lignées et/ou conjoints (pour la garde des enfants, les allers-et-venues école-domicile-lieu de travail, etc.) intervient, selon les cas, lors des situations inextricables et des problèmes organisationnels passagers et impondérables (déplacements, livraisons des animaux, etc.). Ce recours peut se révéler beaucoup plus régulier, voire quotidien, pour les jeunes femmes dont les enfants sont en bas âge.

Pour d'autres exploitantes, les dispositifs professionnels individualisants offrent des possibilités d'auto-organisation des différents temps. Les mobilités exploitation/ famille (garde des enfants en bas âge et soutien scolaire dans le lieu d'exercice professionnel, réalisation des activités administratives et comptables au domicile, etc.)

16. Élodie, 23 ans, père : militaire et mère : auxiliaire de vie, installée en 2012 dans un GAEC : céréales, élevage de veaux, collectif de travail : son beau-père (mi-temps) et son conjoint. 
peuvent avoir l'avantage d'élargir les flexibilités temporelles. Elles permettent un remaniement de l'organisation des activités agricoles et domestiques. À partir du moment où les jeunes femmes parviennent à mieux gérer les différentes temporalités, elles s'investissent dans d'autres activités agricoles en dehors des entreprises (vente directe, etc.). Ces possibilités organisationnelles ne jouent pas en leur faveur dans le sens où elles contribuent à légitimer l'exclusivité masculine des structures collectives.

\section{Une distanciation des femmes par rapport au domestique}

D'autres systèmes organisationnels dans les exploitations ont un effet émancipateur des exploitantes du domestique ${ }^{17}$. Dans les modèles de la division sexuelle des travaux agricoles individualisés, des déclinaisons apparaissent aussi bien du côté des femmes que des hommes. Le contexte global du système exploitation/famille est celui des tentatives d'ajustement, induisant une remise en cause de l'assignation des femmes au domestique. La sphère professionnelle est légitimement un espace de réalisation pour les hommes et les femmes. Dans l'espace privé, les conjoints soutiennent l'engagement professionnel des jeunes exploitantes. Dans les faits, cela passe par leur prise en charge des tâches domestiques et des obligations familiales dans des tranches horaires spécifiques. Dans les modèles sous-tendus par la symétrie des systèmes d'activité des hommes et des femmes, l'obligation d'un partage des tâches domestiques entre conjoints demeure une norme. C'est la condition affichée $\mathrm{du}$ respect du droit de chacun d'exercer une " profession prenante " et de l'engagement affectif. Au vu des situations dans lesquelles ces couples se trouvent (surinvestissement professionnel, gestion de collectifs de travail, etc.), la nécessité dicte également cette répartition égalitaire.

Dans ces deux configurations conjugales, les catégorisations du féminin et du masculin peuvent perdre de leur efficacité opératoire lorsque l'on considère le contenu des activités domestiques. L'investissement des conjoints dans la sphère domestique intervient souvent en appui à une externalisation de la garde des enfants en bas âge et/ou des tâches domestiques (ménage, repassage, etc.). Le processus de légitimation du surinvestissement professionnel des jeunes exploitantes est en œuvre. Leur forte implication dans la sphère productive et les limitations strictes des tâches domestiques consistent à éviter les enchevêtrements des engagements professionnels et domestiques pour s'écarter des " habitudes familiales ». Les spécificités de leurs dispositifs professionnels (recours à des salariés agricoles, articulation du travail collectif et du travail individuel, diversification des systèmes d'action, etc.) les conduisent à impulser de nouvelles dynamiques à la fois professionnelles et familiales. Les caractéristiques de leur rapport à leur entreprise les placent objectivement

17. Ce n'est pas forcément au sein des couples sans enfant que nous retrouvons les prémisses du dépassement de l'assignation féminine au domestique, mais plutôt chez les femmes qui connaissent des expériences familiales singulières. 
et subjectivement du côté de la construction de modèles d'activité originaux marqués par des mobilités de genre.

Le désengagement professionnel des femmes au sein des structures collectives demeurait au cœur de l'ethos agricole jusqu'alors centré sur une hiérarchie de genre. La distanciation de ces jeunes exploitantes par rapport à cet ethos fait rupture avec les enjeux sexués de la conciliation vie professionnelle et vie familiale. Rupture enfin par rapport à leur rôle féminin, pour une plus grande dissociation des places occupées dans les sphères productives et domestiques et un retranchement de leurs logiques entrepreneuriales.

\section{Conclusion}

Les formulations classiques considéraient que les mutations structurelles, et plus particulièrement les "modèles techniques d'exploitation " étaient déterminants dans la transformation des rapports familiaux de production (Barthez, 1982 ; Rieu, 1986). Au terme de notre analyse, nous pouvons soutenir qu'il ne suffit pas de partager un même statut professionnel et un même espace-temps de travail pour se rapprocher d'une indifférenciation des conditions d'exercice entre les femmes et les hommes.

L'analyse des situations de travail individualisées, collectives et isolées permet d'interroger l'efficacité ou non des processus organisationnels au sein du système exploitation/famille. L'individualisation du travail agricole, en tant que condition liée aux positions objectives occupées dans les différents systèmes d'activité, peut freiner l'inscription sociale des femmes dans les processus collectifs d'émancipation au travail. Son dépassement favorise la reconnaissance de leurs contributions et de leurs maîtrises professionnelles pour les travaux individualisés et collectifs, ainsi que l'identification de leurs logiques entrepreneuriales à partir de leur propre vécu socialisé. Les profils présentés permettent de comprendre que les rapports de travail entre les sexes peuvent se trouver clivés. Les divisions du travail agricole profondément marquées par des différenciations sexuées conduisent à une reproduction des fondements de l'exclusion des femmes des processus collectifs de rationalisation et des filières de décision des entreprises agricoles. Les contraintes apparentées à la sexuation des systèmes d'activité s'ajoutent à celles de la sphère domestique où les assignations familiales tendent à invalider le surinvestissement professionnel des exploitantes dans les structures collectives. Les interrelations entre les logiques individuelles et collectives de l'organisation du travail agricole peuvent changer la tendance lorsque les femmes et les hommes mettent en pratique des processus de rationalisation qui tiennent compte des expériences de travail de chacun. Des nouveaux rapports de production en découlent et dessinent d'autres rapports sociaux dans l'espace privé. Ces chemins empruntés conduisent vers un alignement des normes et des pratiques professionnelles et familiales des femmes et des hommes. 


\section{Bibliographie}

Agreste-Recensement agricole, 2012. Exploitations agricoles et superficie agricole utilisée par statut juridique, ministère de l'Agriculture, de l'Agroalimentaire et de la Forêt.

Barthez, A., 1982. Famille, travail et agriculture, Paris, Economica.

Cardon, P., Pfefferkorn, R., Kergoat, D., 2009. L'individuel, le collectif et les rapports sociaux de sexe, in Kergoat, D., Pfefferkorn, R., Cardon, P. (Eds.), Chemins de l'émancipation et rapports sociaux de sexe, Paris, La Dispute, 11-47.

Dahache, S., 2012. La féminisation de l'enseignement agricole. Sociologie des rapports de genre dans le champ des formations professionnelles, Paris, L'Harmattan.

Dahache, S., 2013a. L'évolution de la place des femmes dans l'agriculture. Point de départ éventuel de nouvelles dynamiques professionnelles, Cahiers d'études Le DEMETER 2013 - Économie et Stratégies agricoles, 317-330.

Dahache, S., 2013b. Les filles minoritaires de l'enseignement agricole en France : analyse sociologique d'un dispositif d'aide à l'insertion (sociale, scolaire et professionnelle), Rapport pour le ministère des Sports, de la Jeunesse, de l'Éducation populaire et de la Vie associative, Paris.

Dahache, S., 2014, L'enseignement agricole comme espace de reconfiguration du genre, Nouvelles Questions Féministes, 33, 1, 35-49.

Dufour, A., Giraud, C., 2012. Le travail dans les exploitations d'élevage bovin laitier est-il toujours conjugal ? Inra Productions animales, 25, 167-180.

Giraud, C., Rémy, J., 2008. Les choix des conjoints en agriculture, Revue d'Études en Agriculture et Environnement, 88, 3, 21-46.

Hervieu, B., Purseigle, F., 2013. Sociologie des mondes agricoles, Paris, Armand Colin.

Kergoat, D., 2000. Division sexuelle du travail et rapports sociaux de sexe, in Hirata, H., Laborie, F., Le Daoré, H., Sénotier D. (Eds.), Dictionnaire Critique du Féministe, Paris, Presses Universitaires de France, 35-44.

Lada, E., 2009. Individualisation et isolement au travail dans l'hôtellerie, in Kergoat, D., Pfefferkorn, R., Cardon, P. (Eds.), Chemins de l'émancipation et rapports sociaux de sexe, Paris, La Dispute, 171-194.

Lagrave, R.-M. (Eds.), 1987. Celles de la terre. Agricultrice : l'invention politique d'un métier, Paris, Éditions de l'EHESS.

MSA, 2012. Les femmes dans l'agriculture au $1^{\text {er }}$ janvier 2010, Enquête, Paris.

Muller, P., Faure, A., Gerbaux, F. (Eds.), 1989. Les entrepreneurs ruraux. Agriculteurs, artisans, commerçants, élus locaux, Paris, L'Harmattan.

Rattin, S., 2006. Les femmes accèdent lentement à des statuts plus avantageux : en agriculture, la parité n'est pas de mise, Agreste Primeur, 175.

Rémy, J., 2013. L'exploitation agricole : une institution en mouvement, Cahiers d'études - Le DEMETER 2013 - Économie et Stratégies agricoles, 361-381. 
Rieu, A., 1986. Agricultrices et professionnalisation : une enquête en Midi-Pyrénées, Revue Géographique des Pyrénées et du Sud Ouest, 57, 1, 89-101.

Rieu, A., 2005. Agricultrice : un métier masculin investi par les femmes, in Guichard-Claudic, Y., Kergoat, D., Vilbrod A. (Eds.), L'inversion du genre. Quand les métiers masculins se conjuguent au féminin et réciproquement, Rennes, Presses Universitaires de Rennes, 307-325. 